\title{
Feline vector-borne pathogens in the north and centre of Portugal
}

\author{
Hugo Vilhena ${ }^{1,2+}{ }^{2}$ Verónica L Martinez-Díaz ${ }^{3+}$, Luís Cardoso ${ }^{4,5^{*}}$, Lisete Vieira ${ }^{4}$, Laura Altet ${ }^{6}$, Olga Francino ${ }^{6}$, \\ Josep Pastor ${ }^{7}$ and Ana C Silvestre-Ferreira ${ }^{4}$
}

\begin{abstract}
Background: In recent years, several clinical cases and epidemiological studies of feline vector-borne diseases (FVBD) have been reported worldwide. Nonetheless, information on FVBD agents and their prevalence in Portugal is scarce.

Methods: Three-hundred and twenty domestic cats presented to 30 veterinary medical centres in the north and centre regions of Portugal were randomly sampled. Blood was assayed by real-time polymerase chain reaction (PCR) for genera Anaplasma/Ehrlichia, genus Babesia, Hepatozoon canis, Hepatozoon felis, Leishmania infantum and the genus Rickettsia. Babesia-positive samples were further tested for Babesia canis and Babesia vogeli.

Results: Eighty (25.0\%) out of the 320 cats were positive to at least one vector-borne agent, including seven (2.2\%) cats co-infected with two agents. Two cats (0.6\%) were infected with Anaplasma/Ehrlichia spp., four (1.3\%) with B. canis, 26 (8.1\%) with B. vogeli, 50 (15.6\%) with H. felis, one (0.3\%) with L. infantum and four (1.3\%) with Rickettsia spp. No cat tested positive for $H$. canis. One cat $(0.3 \%)$ was co-infected with $B$. canis and B. vogeli, three (0.9\%) with $B$. vogeli and $H$. felis, one (0.3\%) with $H$. felis and L. infantum, and two (0.6\%) with $H$. felis and Rickettsia spp.

Conclusions: A considerable prevalence of infection with vector-borne pathogens among the domestic feline population of the north and centre of Portugal has been revealed by the present study. Additionally, this is the first detection of $B$. vogeli in cats from Europe and of $H$. felis in cats from Portugal.
\end{abstract}

Keywords: Cats, Feline vector-borne diseases, PCR, Portugal

\section{Background}

Vector-borne diseases compromise a variety of infectious illnesses caused by several agents, including viruses, bacteria, protozoa and helminthes, which are transmitted by ticks, fleas, mosquitoes and phlebotomine sand flies $[1,2]$. Many of these agents are emerging or re-emerging pathogens [3] and some of them are of zoonotic concern $[4,5]$. The frequency of some vector-borne diseases is increasing in Europe, partially due to climatic alterations that have a direct impact on the abundance, geographical distribution and vectorial capacity of arthropod vectors, but also due to the increased mobility of human

\footnotetext{
* Correspondence: Icardoso@utad.pt

${ }^{\dagger}$ Equal contributors

${ }^{4}$ Department of Veterinary Sciences, School of Agrarian and Veterinary Sciences, Universidade de Trás-os-Montes e Alto Douro, Vila Real, Portugal ${ }^{5}$ Parasite Disease Group, Instituto de Biologia Molecular e Celular (IBMC), Universidade do Porto, Oporto, Portugal

Full list of author information is available at the end of the article
}

beings and animals, which further promote the circulation and exchange of vectors and infectious agents $[1,3,6,7]$.

Although several vector-borne agents cause morbidity and mortality in the domestic feline population [8], the importance of some of them as a cause of disease has not yet been clearly determined [9]. This lack of knowledge, associated with the unawareness of the distribution and ecology of feline vector-borne diseases (FVBD) of zoonotic concern, has impaired the implementation of effective control measures to prevent infection of cats, other animals and human beings [1].

The diagnosis of infectious diseases in cats may be challenging, as some infections can occur in healthy cats [10], and whenever present clinical signs are frequently non-specific $[1,11]$. Most of the agents are often present in low numbers in peripheral blood, are difficult to cultivate in vitro, elicited specific antibody responses may be 
inconsistent [11], and serological cross-reactivity can exist between some organisms [12]. Many problems of serology are circumvented by the use of the polymerase chain reaction (PCR) $[12,13]$. In addition to accurate detection of infectious agents in animals, human beings and arthropod vectors, DNA-based techniques allow species characterization of different pathogens, which can be important for treatment and prognosis [6]. Compared with the conventional method, real-time PCR can have a higher sensitivity in some diseases [14] and is a useful tool both for diagnosis and treatment monitoring [13].

Previous clinical case reports [15], and serological or molecular surveys [16-19] have described infection with different vector-borne organisms in cats from Portugal. Nonetheless, information about agents of FVBD and their prevalence in Portugal is scarce. The aims of the present study were to identify the presence and prevalence of vector-borne agents from genera Anaplasma, Babesia, Ehrlichia, Hepatozoon, Leishmania and Rickettsia in cats from the north and centre regions of Portugal, by means of real-time PCR, and to identify risk factors associated with infection.

\section{Methods}

\section{Cats and samples}

Three-hundred and twenty domestic cats from the north $(\mathrm{n}=140)$ and centre $(\mathrm{n}=180)$ of Portugal were randomly sampled in 30 veterinary medical centres, without inclusion/exclusion criteria or pre-established minima/maxima. The number of cats sampled per centre ranged from 1 to 58. This study was ethically approved by the board of the University of Trás-os-Montes e Alto Douro veterinary teaching hospital as complying with the Portuguese legislation for the protection of animals (Law no. 92/1995, from September the 12th).

Whole blood samples were obtained by jugular or cephalic venipuncture into EDTA tubes and stored at $-20^{\circ} \mathrm{C}$ until DNA extraction. Whenever available, medical and geographic data of each cat was collected, including gender, age, breed, living conditions, clinical status, and feline immunodeficiency virus (FIV)/feline leukaemia virus (FeLV) infections status (Table 1). Practitioners classified the cats as clinically suspect, if they had clinical signs compatible with a FVBD, or non-suspect, when they were apparently healthy or had clinical signs not compatible with an infectious disease. Compatible physical signs and clinicopathological abnormalities comprised anorexia, weight loss, gastrointestinal alterations, anemia, thrombocytopenia, leukocytosis or leukopenia, jaundice and dermatological or ocular manifestations without any other attributable aetiology.

\section{DNA extraction, PCR amplification and sequencing}

DNA was obtained from $0.5 \mathrm{ml}$ of peripheral blood, as previously described [14].

Real-time quantitative (q) PCR for Leishmania infantum was carried out according to the method described by Francino et al. [14]. The targets of primers (Table 2) and TaqMan-MGB probes were conserved regions of the kinetoplastic minicircle of L. infantum.

For the other agents, samples were submitted to different qPCR for genera Anaplasma/Ehrlichia, genus Babesia, Hepatozoon canis, Hepatozoon felis and genus Rickettsia. Babesia-positive samples were further tested for Babesia canis and Babesia vogeli. All primers were designed by The Molecular Genetics Veterinary Service, (Universitat Autònoma de Barcelona) (Table 2). Specificity of each primer was: (i) tested in silico, to avoid cross-amplification with other FVBD agents, using sequence information available in Genbank and RDP II databases; and (ii) also validated by the amplification of the positive control for which the PCR had been designed and by the absence of amplification in samples positive to other pathogens; and (iii) by DNA sequencing of some positive samples to confirm agents $B$. canis $(\mathrm{n}=1)$, B. vogeli $(\mathrm{n}=3)$ and $H$. felis $(\mathrm{n}=4)$.

For all agents, qPCR amplification was carried out in a final volume of $20 \mu \mathrm{l}$ using FastStart Universal SYBR Green Master (Roche), $4 \mu$ l of diluted DNA and a final primer concentration depending on the amplified pathogen (Table 2). Thermal cycling profile was $50^{\circ} \mathrm{C}$ for $2 \mathrm{~min}$ and $95^{\circ} \mathrm{C}$ for $10 \mathrm{~min}$, followed by 40 cycles at $95^{\circ} \mathrm{C}$ for $15 \mathrm{~s}$ and $60^{\circ} \mathrm{C}$ for $1 \mathrm{~min}$. Specificity assessment of $\mathrm{qPCR}$ was performed by adding a dissociation curve analysis at the end of the run. The internal reference for cat genomic DNA was the eukaryotic 18S RNA Pre-Developed TaqMan Assay Reagents (Applied Byosystems), which ensured proper qPCR amplification of each sample and that negative results corresponded to true negative samples rather than to a problem with DNA loading, sample degradation or PCR inhibition. Positive qPCR controls were obtained from clinical samples previously amplified and sequenced to confirm the pathogen. Water was used as a negative control. The product of the realtime PCR was sequenced with the BigDye Terminator Cycle Sequencing Ready Reaction Kit (AB, Live Technologies) using the same primers. Sequences obtained were compared with GenBank database (www.ncbi. nlm.nih.gov/BLAST).

\section{Statistical analysis}

Prevalences of infection relative to the independent variables (i.e. gender, age, breed, housing conditions, FeLV/FIV infections and clinical status) were compared by Chi-square or Fisher's exact tests. A $p$ value $<0.05$ was considered as 
Table 1 Prevalence of infection with vector-borne pathogens in cats from the north and centre of Portugal, as determined by PCR

\begin{tabular}{|c|c|c|c|c|c|c|c|c|}
\hline \multirow{2}{*}{$\begin{array}{l}\text { Independent variable/ } \\
\text { category }\end{array}$} & \multirow{2}{*}{$\begin{array}{l}\text { No. of cats } \\
\text { tested (\%) }\end{array}$} & \multicolumn{7}{|c|}{ No. of positive cats (\%) } \\
\hline & & Anaplasma/Ehrlichia & B. canis & B. vogeli & H. felis & L. infantum & Rickettsia & $\geq 1$ agent(s) \\
\hline Gender & 320 & & & & & & & \\
\hline Female & $142(44.4)$ & $2(1.4)$ & $2(1.4)$ & $14(9.9)$ & $19(13.4)$ & $1(0.7)$ & $0(0.0)$ & $36(25.4)$ \\
\hline Male & $178(55.6)$ & $0(0.0)$ & $2(1.1)$ & $12(6.7)$ & $31(17.4)$ & $0(0.0)$ & $4(2.2)$ & $44(24.7)$ \\
\hline Age (years) & 312 & & & & & & & \\
\hline$[0.4-1.5]$ & $90(28.8)$ & $0(0.0)$ & $2(2.2)$ & $11(12.2)^{\mathrm{a}}$ & $13(14.4)$ & $0(0.0)$ & $0(0.0)$ & $23(25.6)$ \\
\hline$[2.0-6]$ & $157(50.3)$ & $1(0.6)$ & $2(1.3)$ & $13(8.3)$ & $25(15.9)$ & $1(0.6)$ & $3(1.9)$ & $42(26.8)$ \\
\hline$[7-20]$ & 65 (20.8) & $1(0.6)$ & $0(0.0)$ & $1(1.5)^{\mathrm{a}}$ & $11(16.9)$ & $0(0.0)$ & $1(0.6)$ & $14(21.5)$ \\
\hline Breed & 320 & & & & & & & \\
\hline DSH & $274(85.6)$ & $2(0.7)$ & $4(1.5)$ & $19(6.9)$ & $43(15.7)$ & $1(0.4)$ & $3(1.1)$ & $66(24.1)$ \\
\hline Pure breed & $46(14.4)$ & $0(0.0)$ & $0(0.0)$ & $7(15.2)$ & $7(15.2)$ & $0(0.0)$ & $1(2.2)$ & $14(30.4)$ \\
\hline Housing & 316 & & & & & & & \\
\hline Totally indoors & $124(39.2)$ & $0(0.0)$ & $0(0.0)$ & $7(5.6)$ & $20(16.1)$ & $0(0.0)$ & $2(1.6)$ & $26(21.0)$ \\
\hline Outdoors access & $192(60.8)$ & $2(1.0)$ & $4(2.1)$ & $19(9.9)$ & $30(15.6)$ & $1(0.5)$ & $2(1.0)$ & $54(28.1)$ \\
\hline FeLV & 117 & & & & & & & \\
\hline Negative & 107 (91.5) & $0(0.0)$ & $1(0.9)$ & $9(8.4)$ & $15(14.0)$ & $0(0.0)$ & $2(1.9)$ & $24(22.4)$ \\
\hline Positive & $10(8.5)$ & $0(0.0)$ & $0(0.0)$ & $1(10.0)$ & $0(0.0)$ & $0(0.0)$ & $0(0.0)$ & $1(10.0)$ \\
\hline FIV & 117 & & & & & & & \\
\hline Negative & $97(82.9)$ & $0(0.0)$ & $1(1.0)$ & $10(10.3)$ & $13(13.4)$ & $0(0.0)$ & $2(2.1)$ & $23(23.7)$ \\
\hline Positive & $20(17.1)$ & $0(0.0)$ & $0(0.0)$ & $0(0.0)$ & $2(10.0)$ & $0(0.0)$ & $0(0.0)$ & $2(10.0)$ \\
\hline Clinical status & 300 & & & & & & & \\
\hline Non-suspect & $132(44.0)$ & $2(1.5)$ & $2(1.5)$ & $16(12.1)$ & $17(12.9)$ & $1(0.8)$ & $1(0.8)$ & $34(25.8)$ \\
\hline Suspect & $168(56.0)$ & $0(0.0)$ & $2(1.2)$ & $10(6.0)$ & 33 (19.6) & $0(0.0)$ & $3(1.8)$ & $46(27.4)$ \\
\hline Total & $320(100)$ & $2(0.6)$ & $4(1.3)$ & $26(8.1)$ & $50(15.6)$ & $1(0.3)$ & $4(1.3)$ & $80(25.0)$ \\
\hline
\end{tabular}

${ }^{a} p=0.031 ;$ B. canis: Babesia canis; B. vogeli: Babesia vogeli; DSH: domestic short-haired; FeLV: feline leukaemia virus; FIV: feline immunodeficiency virus; H. felis: Hepatozoon felis; L. infantum: Leishmania infantum; PCR: polymerase chain reaction.

statistically significant. Analyses were performed with SPSS $^{\circledR} 11.5$ software for Windows (SPSS Inc).

\section{Results}

Table 1 displays data on the prevalence of infection with vector-borne agents among the 320 cats assessed in this study. Cats $(\mathrm{n}=312)$ were aged from 5 months to 20 years (mean: 4.32 years \pm 3.88 standard deviation). Pure breed cats were mainly Persians and Siamese. Absolute numbers and proportions (positive/tested cats) of single and co-infections are shown in Table 3.

Eighty cats $(25.0 \%)$ were qPCR positive to at least one of the tested agents, including seven (2.2\%) cats coinfected with two agents. Two cats $(0.6 \%)$ were infected with Anaplasma/Ehrlichia spp., four (1.3\%) with $B$. canis, 26 (8.1\%) with B. vogeli, 50 (15.6\%) with $H$. felis, one $(0.3 \%)$ with $L$. infantum and four $(1.3 \%)$ with Rickettsia spp. No cat tested positive to $H$. canis. One cat $(0.3 \%)$ was co-infected with $B$. canis and B. vogeli, three $(0.9 \%)$ with $B$. vogeli and $H$. felis, one $(0.3 \%)$ with $H$. felis and L. infantum, and two (0.6\%) with $H$. felis and Rickettsia spp.

Sequencing confirmed B. canis in the one cat $(100 \%$ relatedness to GenBank HQ662634.1), B. vogeli in the three cats (100\% relatedness to GenBank JX871885.1) and $H$. felis in the four cats $(100 \%$ relatedness to GenBank JQ867388.1) whose PCR products were sequenced. Because of the small quantities of amplified bacterial DNA in the genus-specific PCR, additional species-specific PCR assays or DNA sequencing were not performed for those samples positive to genera Anaplasma/Ehrlichia and Rickettsia.

A statistically significant difference $(p=0.031)$ was found between the prevalence of infection with $B$. vogeli in cats aged 5-18 months (0.42-1.5 years) and in cats aged $7-20$ years old (12.2\% versus $1.5 \%$, respectively) (Table 1). No other significant differences were found for the prevalence of infection with each vector-borne agent, at least one agent ( $\geq 1$ agent $[\mathrm{s}]$ ) or co-infections (2 agents), regarding all the independent variables. 
Table 2 Primer sequences of the tested vector-borne pathogens (genera or species)

\begin{tabular}{|c|c|c|c|c|}
\hline Agents & Amplified region & Primer forward $\left(5^{\prime}-3^{\prime}\right)$ & Primer reverse $\left(5^{\prime}-3^{\prime}\right)$ & $\begin{array}{c}\text { Final [primer] } \\
(\mu \mathrm{M})\end{array}$ \\
\hline Anaplasma/Ehrlichia spp. & $16 \mathrm{~S}$ rRNA & GGGTGAGTAATGCRTAGGAATCTACCTAGTA & GGATTATACAGTATTACCCAYCATTTCTARTG & 0.5 \\
\hline Babesia spp. & 18S rRNA & GTGGCTITTCCGATTCGTCG & TTCCTITAAGTGATAAGGTTCACAAAACTT & 0.3 \\
\hline Babesia canis & $18 \mathrm{~S}$ rRNA & CGGTTTGACCATTTGGTTGGTTA & CCATGCTGAAGTATTCAAGACAAAAGT & 0.3 \\
\hline Babesia vogeli & $18 \mathrm{~S}$ rRNA & CATTCGTTTGGCTITITCGAG & CCATGCTGAAGTATTCAAGACAAAAGT & 0.3 \\
\hline Hepatozoon felis & $18 \mathrm{~S}$ rRNA & CTTACCGTGGCAGTGACGGT & TGTTATTTCTTGTCACTACCTCTCTTATGC & 0.3 \\
\hline Leishmania infantum & kinetoplast DNA & AACTITTCTGGTCCTCCGGGTAG & ACCCCCAGTTTCCCGCC & 0.9 \\
\hline Rickettsia spp. & $16 S$ rRNA & AGCCTGATCCAGCAATACCGA & CGGGGCTIITCTGCAAGTAA & 0.3 \\
\hline
\end{tabular}

\section{Discussion}

The present study represents the most comprehensive investigation on FVBD performed in Portugal, in terms of the number of tested cats and extension of the covered geographical area, and reveals a considerable prevalence of infection in domestic cats from the north and centre regions of Portugal. Anaplasma/Ehrlichia spp., $B$. canis, B. vogeli, $H$. felis, L. infantum and Rickettsia spp. were detected among the assayed feline population.

Several ehrlichial and rickettsial infections are shared by man and companion animals [20]. In the present study, Anaplasma/Ehrlichia spp. and Rickettsia spp. DNA were detected in $0.6 \%$ and $1.3 \%$ of the cats, respectively. In cats from southern Portugal, seroprevalences (by immunofluorescence antibody tests) were $13.5 \%$ for Anaplasma phagocytophilum and $18.9 \%$ for Rickettsia conorii [17], and 26.3\% for R. conorii/Rickettsia felis [19]. Furthermore, Breu et al. [19] also reported feline infection by Ehrlichia canis in Portugal. A national serological study on canine vector-borne diseases in Portugal detected a significantly higher seroprevalence of antibodies to Anaplasma spp. and E. canis in dogs from southern Portugal, when compared to dogs from the northern and central regions [7].

The present study represents the first report on the prevalence of Babesia spp. in cats from Portugal. A higher prevalence of Babesia spp. was found in Portuguese cats (9.4\%), in comparison with that detected in cats from Barcelona, Spain (0/100) by Tabar et al. [12]. Interestingly, it can also be presumed that the most prevalent piroplasm in the Portuguese feline population is $B$. vogeli, instead of $B$. canis, which was the piroplasm most frequently detected in dogs with babesiosis from the north of Portugal [21]. So far, feline infection with $B$. vogeli has only been described in cats from Trinidad, Trinidad and Tobago [22], and Bangkok, Thailand [23]. To the best of our knowledge, this is the first time that infection with $B$. vogeli has been detected in cats from Europe.

Feline co-infections with other erythrocytic pathogens such as Mycoplasma spp., Cytauxzoon felis or other species of Babesia may be possible [24,25]. In the present study, only one cat was found co-infected with B. canis and B. vogeli out of the 29 cats infected with Babesia spp. Infection with B. canis and/or the Babesia microtilike piroplasm (syn. Theileria annae) was previously described in three cats from Portugal [26], but no information is available on the geographical origin of those cats. The $B$. microti-like piroplasm has also recently been found in dogs from northern Portugal affected by babesiosis [27]. In the present study, other species of the genera Babesia and Theileria were not assessed. Nevertheless, as the entire results positive to the genus Babesia had an assigned species (i.e. B. vogeli, B. canis or both), although not impossible, a co-infection with the B. microti-like piroplasm seems unlikely.

Age has been described as a predisposing factor for feline infection with Babesia spp., with younger cats (less than 3 years old) more predisposed to infection in endemic areas $[24,28]$, and older cats more susceptible to the disease following relocation to an endemic area or in conjunction with concurrent disease, immunosuppression or severe trauma [24]. In the present study, juvenile cats ( $\leq 1.5$ year) had a significantly higher prevalence of $B$. vogeli infection

Table 3 Prevalence of single and co-infections with vector-borne pathogens in $\mathbf{3 2 0}$ cats from north and centre Portugal, as determined by PCR

\begin{tabular}{lll}
\hline Agent(s) & No. of positive cats & $\%$ \\
\hline Single infections & 73 & 22.8 \\
Anaplasma spp./Ehrlichia spp. & 2 & 0.6 \\
B. canis & 3 & 0.9 \\
B. vogeli & 22 & 6.9 \\
H. felis & 44 & 13.8 \\
L. infantum & 0 & 0.0 \\
Rickettsia spp. & 2 & 0.6 \\
Co-infections & 7 & 2.2 \\
B. canis + B. vogeli & 1 & 0.3 \\
B. vogeli + H. felis & 3 & 0.9 \\
H. felis + L. infantum & 1 & 0.3 \\
H. felis + Rickettsia spp. & 2 & 0.6 \\
Total & 80 & 25.0 \\
\hline B. cans: Babesa canis: B. vogel: Babesa & . &
\end{tabular}

B. canis: Babesia canis; B. vogeli: Babesia vogeli; H. felis: Hepatozoon felis; L. infantum: Leishmania infantum; PCR: polymerase chain reaction. 
in comparison with geriatric cats ( $\geq 7$ years), probably because of the less mature immune status of young cats.

Infection with Hepatozoon spp. is frequently reported in dogs [29] but not in cats. Furthermore, the Hepatozoon species that infect cats have not been definitely characterized $[30,31]$. Some authors have suggested that $H$. canis is the agent responsible for feline infection, but a new, yet unnamed, species of Hepatozoon genetically distinct from $H$. canis was recently detected in cats from southern and northeastern Spain [30,32]. Infection with H. felis was firstly described by Tabar et al. in cats from Barcelona [12].

This is also the first report of feline infection with $H$. felis in Portugal. Molecularly confirmed infection with $H$. canis in cats from southern Portugal [19] and in a dog from northern Portugal [21] had already been described. The detected $15.6 \%$ prevalence of infection with $H$. felis in the present study is similar to that of Hepatozoon spp. (16\%) described by Ortuño et al. [30] in stray cats from Barcelona ( $p=0.960)$, but higher than the $0.6 \%$ of Hepatozoon spp. found in Spanish domestic cats from a non-identified geographical background [32] and the $4.0 \%$ of $H$. felis in domestic cats from Barcelona [12] $(p<0.001$ and $p=0.002$, respectively). Moreover, the present study sustains the fact that Hepatozoon infection is widespread in the feline population of the Iberian Peninsula. Baneth et al. [33], in a study from Israel, detected that most infected cats were young domestic short-haired males and that there was an overrepresentation of cats with retroviral disease. In the present study, no statistically significant association was found between infection with $H$. felis and independent variables including clinical status and FIV/FeLV infection (Table 1).

Leishmaniasis is an endemic zoonosis prevalent in the Mediterranean basin [34,35]. The increase in the number of infections and disease cases reported in recent years, together with the results described in different prevalence studies, suggest that cats can act as a secondary reservoir host instead of an accidental one in areas where Leishmania spp. are endemic [36-38]. Several surveys of Leishmania spp. infection in cats have been performed in different countries by different techniques, with prevalences ranging between $0 \%$ and $68 \%$ [9,16,18,39-43]. In the present study, detected prevalence $(0.3 \%)$ might have been different if the qPCR was carried out with another tissue sample, such as bone marrow, spleen or liver. Results are lower than the $2.8 \%$ seroprevalence (by the direct agglutination test and an enzyme-linked immunosorbent assay) found in cats from northern Portugal [18] and also lower than the 20.3\% obtained in blood samples from cats of Greater Lisbon (southern-central Portugal) by PCR [16] ( $p=0.01$ and $p<0.001$, respectively). For the appropriate agents under assessment in the present study, comparative differences in the prevalence values can be related to different detection techniques (serology versus molecular analysis) as well as to a different geographical origin of cats (north and centre versus south). The latter may determine differences in climatic conditions, arthropod vector survival and agent transmission rate.

Associations between housing conditions and prevalence of infection were not found among cats for any one of the agents. Conversely, in a comparable study on canine vector-borne diseases, also in Portugal, a significantly higher sero-positivity to at least one agent (i.e. Dirofilaria immitis, E. canis, Borrelia burgdorferi sensu lato, Anaplasma spp. and/or L. infantum) has been found in clinically suspect dogs with an outdoor or mixed lifestyle [7].

\section{Conclusions}

In the north and centre regions of Portugal, a high prevalence of infection with $B$. vogeli and $H$. felis, and a relatively low prevalence of infection with Anaplasmal Ehrlichia spp., B. canis, L. infantum and Rickettsia spp. were found in cats. Further studies on these and other vector-borne agents are needed to better understand their epidemiological and clinical importance. It is also necessary to call on veterinarians and owners to adopt effective control measures, including chemoprophylaxis against the ectoparasite vectors, in order to prevent infection of cats with agents of FVBD and their potential transmission to other domestic and wild animals as well as to human beings.

\section{Competing interests}

The authors declare that they have no competing interests.

\section{Authors' contributions}

HV collected samples and clinical data, performed data analysis and drafted the manuscript; VLM-D performed DNA extraction and molecular analyses; LC co-supervised the study, performed data analysis and revised the manuscript; LV collected samples and clinical data; LA and OF designed the primers, supervised molecular analyses and provided conceptual advice; JP and ACS-F planned and supervised the study, coordinated sample collection and reviewed the manuscript. All authors read and approved the final manuscript.

\section{Acknowledgements}

The authors thank the cooperation of the practitioners and auxiliary staff at the 30 veterinary medical centres that contributed with collection of samples and complementary data. Publication of this article has been sponsored by Bayer Animal Health $\mathrm{GmbH}$.

\section{Author details}

${ }^{1}$ Department of Veterinary Medicine, Escola Universitária Vasco da Gama, Coimbra, Portugal. Baixo Vouga Veterinary Hospital, Águeda, Portugal. ${ }^{3}$ Department of Animal and Food Sciences, Agrigenomics Research Centre (CRAG), Veterinary Faculty, Universitat Autònoma de Barcelona, Bellaterra (Barcelona), Spain. ${ }^{4}$ Department of Veterinary Sciences, School of Agrarian and Veterinary Sciences, Universidade de Trás-os-Montes e Alto Douro, Vila Real, Portugal. ${ }^{5}$ Parasite Disease Group, Instituto de Biologia Molecular e Celular (IBMC), Universidade do Porto, Oporto, Portugal. 'Veterinary Service for Molecular Genetics, Department of Animal and Food Sciences, Veterinary Faculty, Universitat Autònoma de Barcelona, Bellaterra (Barcelona), Spain. ${ }^{7}$ Department of Animal Medicine and Surgery, Veterinary Faculty, Universitat Autònoma de Barcelona, Bellaterra (Barcelona), Spain.

Received: 31 January 2013 Accepted: 6 March 2013

Published: 15 April 2013 


\section{References}

1. Otranto D, Dantas-Torres F: Canine and feline vector-borne diseases in Italy: current situation and perspectives. Parasit Vectors 2010, 3:2

2. Baneth G, Bourdeau P, Bourdoiseau G, Bowman D, Breitschwerdt E, Capell G, Cardoso L, Dantas-Torres F, Day M, Dedet JP, Dobler G, Ferrer L, Irwin P, Kempf V, Kohn B, Lappin M, Little S, Maggi R, Miró G, Naucke T, Oliva G, Otranto D, Penzhorn B, Pfeffer M, Roura X, Sainz A, Shaw S, Shin S, SolanoGallego L, Straubinger R, et al: Vector-borne diseases - constant challenge for practicing veterinarians: recommendations from the CVBD World Forum. Parasit Vectors 2012, 5:55.

3. Beugnet F, Marié J-L: Emerging arthropod-borne diseases of companion animals in Europe. Vet Parasitol 2009, 163:298-305.

4. Shaw S, Day M: Introduction. In Arthropod-Borne Infectious Diseases of the Dog and Cat. Edited by Shaw SE, Day MJ. London: Manson Publishing; 2005:9-10

5. Hii SF, Kopp SR, Thompson MF, O'Leary CA, Rees RL, Traub RJ: Molecular evidence of Rickettsia felis infection in dogs from Northern Territory, Australia. Parasit Vectors 2011, 4:198

6. Cardoso L: Dogs, arthropod-transmitted pathogens and zoonotic diseases. Trends Parasitol 2010, 26:61-62.

7. Cardoso L, Mendão C, Madeira de Carvalho L: Prevalence of Dirofilaria immitis, Ehrlichia canis, Borrelia burgdorferi sensu lato, Anaplasma spp. and Leishmania infantum in apparently healthy and CVBD-suspect dogs in Portugal - a national serological study. Parasit Vectors 2012, 5:62.

8. Eberhardt JM, Neal K, Shackelford T, Lappin MR: Prevalence of selected infectious disease agents in cats from Arizona. J Feline Med Surg 2006, 8:164-168.

9. Ayllón T, Diniz PP, Breitschwerdt EB, Villaescusa A, Rodríguez-Franco F, Sainz A: Vector-borne diseases in client-owned and stray cats from Madrid, Spain. Vector Borne Zoonotic Dis 2012, 12:143-150.

10. Kamrani A, Parreira VR, Greenwood J, Prescott JF: The prevalence of Bartonella, hemoplasma, and Rickettsia felis infections in domestic cats and in cat fleas in Ontario. Can J Vet Res 2008, 72:411-419.

11. Kenny M: Laboratory diagnosis of arthropod-transmitted infections. In Arthropod-Borne Infectious Diseases of the Dog and Cat. Edited by Shaw SE, Day MJ. London: Manson Publishing; 2005:41-50.

12. Tabar MD, Altet L, Francino O, Sánchez A, Ferrer L, Roura X: Vector-borne infections in cats: molecular study in Barcelona area (Spain). Vet Parasitol 2008, 151:332-336

13. Martínez V, Quilez J, Sanchez A, Roura X, Francino O, Altet L: Canine leishmaniasis: the key points for $\mathrm{QPCR}$ result interpretation. Parasit Vectors 2011, 4:57.

14. Francino O, Altet L, Sánchez-Robert E, Rodriguez A, Solano-Gallego L, Alberola J, Ferrer L, Sánchez A, Roura X: Advantages of real-time PCR assay for diagnosis and monitoring of canine leishmaniosis. Vet Parasitol 2006, 137:214-221.

15. Costa-Durão JF, Rebelo E, Peleteiro MC, Correia JJ, Simões G: Primeiro caso de leishmaniose em gato doméstico (Felis catus domesticus) detectado em Portugal (Concelho de Sesimbra). Nota preliminar. Rev Port Cienc Vet 1994, 89:140-144.

16. Maia C, Gomes J, Cristóvão J, Nunes M, Martins A, Rebêlo E Campino L. Feline Leishmania infection in a canine leishmaniasis endemic region, Portugal. Vet Parasitol 2010, 174:336-340.

17. Alves AS, Milhano N, Santos-Silva M, Santos AS, Vilhena M, Sousa R: Evidence of Bartonella spp., Rickettsia spp. and Anaplasma phagocytophilum in domestic, shelter and stray cat blood and fleas, Portugal. Clin Microbiol Infect 2009, 15(Suppl 2):1-3.

18. Cardoso L, Lopes AP, Sherry K, Schallig H, Solano-Gallego L: Low seroprevalence of Leishmania infantum infection in cats from northern Portugal based on DAT and ELISA. Vet Parasitol 2010, 174:37-42

19. Breu D, Menn B, Guthard J, Lorentz S, Naucke T: Hepatozoon canis may be considered a co-infecting pathogen in dogs and cats from Portugal and Sardinia. In Proceedings of the $21^{\text {st }}$ ECVIM-CA Annual Congress: 8-10 September 2011; Seville. Edited by Mandigers PJJ, German AJ. Seville: European College of Veterinary Internal Medicine - Companion Animals; 2011:255.

20. Day MJ: One health: the importance of companion animal vector-borne diseases. Parasit Vectors 2011, 4:49.

21. Cardoso L, Yisaschar-Mekuzas Y, Rodrigues FT, Costa A, Machado J, Diz Lopes D, Baneth G: Canine babesiosis in northern Portugal and molecular characterization of vector-borne co-infections. Parasit Vectors 2010, 3:27.
22. Georges K, Ezeokoli CD, Newaj-Fyzul A, Campbell M, Mootoo N, Mutani A, Sparagano OA: The application of PCR and reverse line blot hybridization to detect arthropod-borne hemopathogens of dogs and cats in Trinidad. Ann N Y Acad Sci 2008, 1149:196-199.

23. Simking P, Wongnakphet S, Stich RW, Jittapalapong S: Detection of Babesia vogeli in stray cats of metropolitan Bangkok, Thailand. Vet Parasitol 2010, 173:70-75

24. Ayoob AL, Prittie J, Hackner SG: Feline babesiosis. J Vet Emerg Crit Care 2010, 20:90-97.

25. Baneth G, Kenny MJ, Tasker S, Anug Y, Shkap V, Levy A, Shaw SE: Infection with a proposed new subspecies of Babesia canis, Babesia canis subsp. presentii, in domestic cats. J Clin Microbiol 2004, 42:99-105.

26. Criado-Fornelio A, Martinez-Marcos A, Buling-Saraña A, Barba-Carretero JC: Presence of Mycoplasma haemofelis, Mycoplasma haemominutum and piroplasmids in cats from southern Europe: a molecular study. Vet Microbiol 2003, 93:307-317.

27. Simões PB, Cardoso L, Araújo M, Yisaschar-Mekuzas Y, Baneth G: Babesiosis due to the canine Babesia microti-like small piroplasm in dogs - first report from Portugal and possible vertical transmission. Parasit Vectors 2011, 4:50.

28. Solano-Gallego L, Baneth G: Babesiosis in dogs and cats - expanding parasitological and clinical spectra. Vet Parasitol 2011, 181:48-60.

29. Otranto D, Dantas-Torres F, Weigl S, Latrofa MS, Stanneck D, Decaprariis D, Capelli G, Baneth G: Diagnosis of Hepatozoon canis in young dogs by cytology and PCR. Parasit Vectors 2011, 4:55

30. Ortuño A, Castellà J, Criado-Fornelio A, Buling A, Barba-Carretero JC: Molecular detection of a Hepatozoon species in stray cats from a feline colony in North-eastern Spain. Vet J 2008, 177:134-135.

31. Baneth G: Perspectives on canine and feline hepatozoonosis. Vet Parasitol 2011, 181:3-11.

32. Criado-Fornelio A, Ruas JL, Casado N, Farias NA, Soares MP, Müller G, Brumt $J G$, Berne ME, Buling-Saraña A, Barba-Carretero JC: New molecular data on mammalian Hepatozoon species (Apicomplexa: Adeleorina) from Brazil and Spain. J Parasitol 2006, 92:93-99.

33. Baneth G, Aroch I Tal N, Harrus S. Hepatozoon species infection in domestic cats: a retrospective study. Vet Parasitol 1998, 79:123-133.

34. Solano-Gallego L, Miró G, Koutinas A, Cardoso L, Pennisi MG, Ferrer L, Bourdeau P, Oliva G, Baneth G: LeishVet guidelines for the practical management of canine leishmaniosis. Parasit Vectors 2011, 4:86.

35. Palatnik-de-Sousa CB, Day MJ: One Health: the global challenge of epidemic and endemic leishmaniasis. Parasit Vectors 2011, 4:197.

36. Simões-Mattos L, Bevilaqua C, Mattos M, Pompeu M: Feline leishmaniasis: uncommon or unknown? Rev Port Cienc Vet 2004, 99:79-87.

37. Silva S, Rabelo P, Gontijo N, Ribeiro R, Melo M, Ribeiro V, Michalick M: Firs report of infection of Lutzomyia longipaldis by Leishmania (Leishmania) infantum from a naturally infected cat of Brazil. Vet Parasitol 2010, 174:150-154.

38. Maia C, Campino L: Can domestic cats be considered reservoir hosts of zoonotic leishmaniasis? Trends Parasitol 2011, 27:341-344.

39. Poli A, Abramo F, Barsotti P, Leva S, Gramiccia M, Ludovisi A, Mancianti F: Feline leishmaniosis due to Leishmania infantum in Italy. Vet Parasitol 2002, 106:181-191.

40. Pennisi MG: A high prevalence of of feline leishmaniasis in Southern Italy In Canine Leishmaniasis: moving towards a solution. Edited by Killick-Kendrick R. Boxmeer: Intervet; 2002:39-48.

41. Diakou A, Papadopoulos E, Lazarides K: Specific anti-Leishmania spp. antibodies in stray cats in Greece. J Feline Med Surg 2009, 11:728-730.

42. Bresciani KD, Serrano AC, Matos LV, Savani ES, D'Auria SR, Perri SH, Bonello FL, Coelho WM, Aoki CG, Costa AJ: Ocorrência de Leishmania spp. em felinos do município de Araçatuba, SP. Rev Bras Parasitol Vet 2010, 19:127-129.

43. Millán J, Zanet S, Gomis M, Trisciuoglio A, Negre N, Ferroglio E: An investigation into alternative reservoirs of canine leishmaniasis on the endemic island of Mallorca (Spain). Transbound Emerg Dis 2011, 58:352-357.

doi:10.1186/1756-3305-6-99

Cite this article as: Vilhena et al:: Feline vector-borne pathogens in the north and centre of Portugal. Parasites \& Vectors 2013 6:99 